\title{
Integrative Analysis Reveals a miRNA-mRNA Regulatory Network and Potential Causative Agents in the Asthmatic Airway Epithelium
}

\author{
Jintao Zhang ${ }^{1, *}$ \\ Zihan Wang ${ }^{l}, *$ \\ Dong Zhang' \\ Yun Pan' \\ Xiaofei Liu' \\ Xinrui Qiao' \\ Wenjing Cui ${ }^{\prime}$ \\ Liang Dong ${ }^{1,2}$
}

'Department of Respiratory, Shandong Qianfoshan Hospital, Cheeloo College of Medicine, Shandong University, Jinan,

People's Republic of China; ${ }^{2}$ Department of Respiratory, Shandong Provincial Qianfoshan Hospital, Shandong University, The First Affiliated Hospital of Shandong First Medical University, Shandong Institute of Respiratory Diseases, Jinan, People's Republic of China

*These authors contributed equally to this work
Correspondence: Liang Dong Department of Respiratory, Shandong Provincial Qianfoshan Hospital, Shandong University, The First Affiliated Hospital of Shandong First Medical University, Shandong Institute of Respiratory Diseases, Jinan, People's Republic of China Emaild15506@126.com
Background: During asthma progression, the intricate molecular networks, including microRNA (miRNA) transcriptional regulation in airway epithelium, remain largely undefined. The abnormal expression of miRNAs in asthmatic airway epithelium is a recent and fast-growing area in developing diagnostic and therapeutic targets for asthma.

Material and Methods: Analyses were conducted to compare airway epithelial miRNAs and gene expression between patients with asthma and healthy subjects from three datasets (two for miRNAs expression profiles and one for gene expression profile). The interactions network between differentially expressed (DE)-miRNAs and mRNAs was further identified for functional analysis. To verify the involvement and functions of all the identified miRNAs in asthma, we constructed two cellular models of asthma. The most promising causal miRNA candidate, miR-1246, was examined in an in vitro system to explore its targets and roles in asthma pathophysiology.

Results: Through integrative analysis, we obtained six miRNAs with 31 validated target genes in airway epithelium associated with asthma. Next, we confirmed that these miRNAs were all associated with asthma progression by in vitro functional experiments. They may participate in eosinophilic inflammation (miR-92b-3p, miR-1246, miR-197-3p, and miR124-5p) or remodeling (miR-197-3p, miR-193a-5p, miR-1246, and miR-92b-3p). Additionally, some other non-screened valuable miRNAs were also examined and identified (miR-21-5p and miR-19b-3p), and some detected in blood correlated with the disease status. Furthermore, we found that miR-1246 could directly target POSTN and influence epithelialto-mesenchymal transition and fibrosis in airway epithelial cells.

Conclusion: We constructed a preliminary epithelial regulatory network in asthma based on six identified miRNAs and their valuable target genes. Candidate factors in the biological miRNA-mRNA network in airway epithelium may provide further information on the pathogenesis of asthma. Strikingly, among all screened miRNAs, miR-1246, which could interact with POSTN may have multifunctional effects in the course of asthma and be a promising agent for asthma treatment and molecular subtyping.

Keywords: asthma, miRNA, regulatory network, biomarkers, molecular subtyping

\section{Introduction}

Bronchial asthma is a highly prevalent chronic airway disease affecting nearly 300 million people worldwide. ${ }^{1}$ As air pollution has increased, the global incidence of asthma has risen. ${ }^{2}$ Although stepped treatments for asthma have been standardized, some patients still remain symptomatic or show only partial improvement after intensive therapy. ${ }^{3}$ Worse still, because of the limitations in diagnostic 
modalities, many patients do not get a timely diagnosis and proper treatment, resulting in irreversible functional and structural damage to the lungs. ${ }^{4}$ In the era of precision medicine, elucidating the molecular mechanism of asthma pathogenesis and identifying suitable biomarkers for precisely categorizing asthma are urgently needed. ${ }^{5}$

The airway epithelium is the first barrier to pathogens and allergic stimuli in the lungs. ${ }^{6}$ Based on single-cell RNA sequencing, many newly recognized cell populations with unique molecular signatures have been discovered in the airway epithelium. ${ }^{6}$ Importantly, the airway epithelium is also accepted as an active player in immune responses and may play critical roles in the development and progression of asthma. ${ }^{7,8}$ However, knowledge of the details of molecular mechanisms in the airway epithelium in pathological processes is insufficient, thereby hindering effective therapeutic strategies. ${ }^{9}$

MicroRNA (miRNA) is defined as a kind of noncoding RNA with a length of $18-26$ bp. As essential regulators, miRNAs are significantly involved in the development of multiple pathological events and human diseases. ${ }^{10}$ Asthma which is a complex and heterogeneous disease, is influenced by various elements, including miRNAs. ${ }^{11}$ These miRNAs may be involved in the onset and course of asthma and could serve as potential biomarkers to aid in diagnosing of asthma and better phenotypic typing. ${ }^{11}$

Here, we constructed a preliminary asthmatic miRNAmRNA regulatory network in airway epithelium by using bioinformatics analysis. Three microarray datasets (GSE25230, GSE142237, and GSE43696), including miRNA and mRNA expression profile datasets, were studied in bronchial epithelial biopsy samples of patients with and without asthma. Three online tools (TargetScan, miRWalk and TarBase) were used to screened target genes of identified miRNAs. Receiver operating characteristics (ROC), Gene Ontology (GO), Kyoto Encyclopedia of Genes and Genomes (KEGG) pathway enrichment analyses, and protein-protein interaction (PPI) networks were studied to further explore the functions of the identified miRNA and their target genes. Moreover, two major in vitro models of asthma, induced by interleukin (IL)-13 or transforming growth factor (TGF)- $\beta 1$, were used to further characterize the functions of all screened miRNAs and some other miRNAs of interest, among which miR-1246, which regulates the expression of POSTN and remodeling-related genes, might serve as a novel biomarker and target for asthma diagnosis and combined therapy. We also measured the expression of these miRNAs in blood samples of patients to further ascertain their clinical values in asthma. This study may be helpful for elucidating the pathophysiological processes and identifying diagnostic biomarkers for asthma.

\section{Materials and Methods Data Sources}

The data expression profiles of asthma were searched in the Gene Expression Omnibus (GEO) database, and two independent miRNA datasets and one mRNA dataset of research on the airway epithelium in human asthma, GSE25230, GSE142237, and GSE43696, respectively, were selected. Differential miRNAs and mRNAs were screened in airway epithelial samples obtained from biopsy in patients with and without asthma.

\section{Analyses of miRNA-mRNA Targets}

Investigating the target genes of miRNAs is crucial for identifying the regulatory mechanisms and functions of miRNAs. Herein, we identified six potential miRNAs and their target genes via three online tools: Targetscan, miRWalk, and TarBase. The miRNA targets were screened based on the overlapping results from the three websites. Then, the regulatory networks of the miRNA-mRNA pairs were extracted (based on an expression fold change $>2.5$ and a false discovery rate (FDR) $<0.05$ ) and visualized using Cytoscape software (http://cytoscape.org/). To ascertain the active regulatory networks in the miRNA-mRNA pairs, we also downloaded the expression profiling data from GSE43696 (analysis of isolated fresh bronchial epithelial cells obtained by endobronchial biopsy from 20 patients without asthma and 88 patients with asthma).

\section{Receiver Operating Characteristic (ROC) Analysis}

The ROC curves were drawn using the pROC package of R software (version 3.6.2).

\section{Gene Ontology (GO) Annotation and Kyoto Encyclopedia of Genes and Genomes (KEGG) Pathway Enrichment Analyses of the DEGs}

GO annotation and KEGG pathway enrichment analyses were performed using the Database for Annotation, Visualization and Integrated Discovery (DAVID) (selected with enrichment significance evaluated at $p<0.05$ ), which 
revealed the biological processes (BPs), cellular components (CCs), molecular functions (MFs) and pathways associated with the selected miRNAs.

\section{Protein-Protein Interaction (PPI) Network Construction}

To gain insights into the interactions between the identified miRNAs and their target genes, a PPI network was constructed and analysed with the STRING tool to reveal the molecular mechanisms underlying asthma. Target genes in the PPI network served as nodes, the lines between two nodes denoted associated interactions, and the strength of the interaction was expressed by the color of the line. The corresponding interactions were visualized using Cytoscape software.

\section{Cell Culture and Treatment}

The BEAS-2B human bronchial epithelial cells were obtained from Shanghai Fuheng Biology Co., Ltd. and incubated in Dulbecco's modified Eagle's medium containing $10 \%$ fetal bovine serum (Gibco, USA) and a moderate amount of antibiotics. The cells were maintained at $37^{\circ} \mathrm{C}$ in a humidified incubator containing $5 \%$ $\mathrm{CO}_{2}$. To construct an asthma-associated cellular model (airway remodeling/epithelial mesenchymal transition (EMT) or eosinophilic inflammation), we stimulated BEAS-2B cells with human IL-13 protein or human TGF- $\beta 1$ protein (Abbkine Scientific Co., Ltd., China) at various concentrations for the indicated time. BEAS-2B cell transfections were performed using the Interfering ${ }^{\circledR}$ reagent (Polyplus-Transfection SA) according to the manufacturer's instructions. We obtained 293 T cells from the American Type Culture Collection (Manassas, VA, USA) and grew them in DMEM with 10\% FBS. Negative controls and a miR-1246 mimic and inhibitor were synthesized and purchased from Shanghai GenePharma Co., Ltd.

\section{Clinical Sample Collection}

To further explore the value of screened miRNAs in asthma, we recruited 10 healthy control and 10 patients with asthma. The diagnosis of asthma was made according to the Global Initiative for Asthma (GINA) (updated in 2021). Sample collection was approved by the Shandong Qianfoshan Hospital Affiliated to Shandong University; all patients provided written informed consent and the characteristics of subjects are shown in Table S1. A $500 \mu \mathrm{L}$ blood sample was used for RNA extraction and detection of level miRNA expression. Research was conducted accordance with Helsinki declaration.

\section{RNA Isolation and RT-qPCR Analysis}

Total RNA was extracted from the plasma sample of patients or BEAS-2B cells with the RNAex Pro Reagent (Accurate Biotechnology Co., Ltd., China) according to the manufacturer's instructions. After quantification using spectrophotometry, $2 \mu \mathrm{g}$ of RNA was used to synthesize cDNA by using a reverse transcription kit (TOYOBO Co., Ltd., Japan). Reverse transcription-quantitative PCR (RTqPCR) was performed using SYBR Green PCR Master Mix (Accurate Biotechnology Co., Ltd., China). The miRNA primers are shown in Table 1.

\section{Immunoblotting}

Total cellular proteins were extracted by using RIPA and then subjected to Western blot experiments for quantification. In order to carry out Western blot experiments, total proteins were segregated using sodium-dodecyl sulfate polyacrylamide gel electrophoresis and transferred onto polyvinylidene difluoride membranes. After briefly washing, Tris-buffered saline with 1\% Tween 20 containing 3\% bovine serum albumin (BSA) was used for membrane blocking for $1 \mathrm{~h}$. Then, the membranes were incubated with the primary antibodies including periostin (1:1000, ab152099, Abcam, USA) and GAPDH (1:1000, BA2913, Boster Biological Technology, China) overnight at $4{ }^{\circ} \mathrm{C}$. The following day, the membranes were removed with tweezers and then washed in TBST three times and incubated with horseradish peroxidase (HRP)conjugated goat anti-rabbit $\operatorname{IgG}(\mathrm{H}+\mathrm{L}) \quad(1: 3000$, GB23303, Servicebio, China) at room temperature for 1 h. After washing with TBST again, the membranes were subjected to chemiluminescence by using an enhanced chemiluminescence substrate.

\section{Immunofluorescence}

BEAS-2B cells were plated on cell climbing slices in a 24well plate. Following the designated treatments, cell climbing slices were washed with phosphate-buffered saline (PBS), fixed with $4 \%$ paraformaldehyde, and then incubated with $0.5 \%$ triton X-100 for permeabilization. Then, the cells were blocked with $1 \%$ BSA for $1 \mathrm{~h}$ at room temperature. The primary anti-periostin antibody (1:200, ab152099, Abcam, USA), anti-fibronectin antibody (1:200, JF0582, Huabio, China), and anti-COL1A1 antibody (1:200, BA0325, Boster, China) were used to 
Table I Primers for qRT-PCR

\begin{tabular}{|c|c|c|}
\hline Symbol & Primer Sequence $\left(5^{\prime}-3^{\prime}\right)$ & Size \\
\hline hsa-miR-197-3p & $\begin{array}{l}\text { Forward: TTCAGTTCACCACCTTCTCCA } \\
\text { Reverse: TATCCTTCTTGACGACTCCTTGAC }\end{array}$ & $73 \mathrm{bp}$ \\
\hline hsa-miR-I24-5p & $\begin{array}{c}\text { Forward: ATCCTATCGTGTTCACAGCG } \\
\text { Reverse: TATGGTTGTTCACCTCTCGTTCAC }\end{array}$ & $73 \mathrm{bp}$ \\
\hline hsa-miR-I246 & $\begin{array}{c}\text { Forward: TTCGACGTGAATGGATTTTTG } \\
\text { Reverse: TATCGTTGTACTCCAGACCAAGAC }\end{array}$ & $68 \mathrm{bp}$ \\
\hline hsa-miR-92b-3p & $\begin{array}{l}\text { Forward: GTAGCATCTGGTATTGCACTCGTC } \\
\text { Reverse: TATGGTTGTTGACGACTGGTTGAC }\end{array}$ & $73 \mathrm{bp}$ \\
\hline hsa-miR-193a-5p & $\begin{array}{c}\text { Forward: ACGCTGGGTCTTTGCGG } \\
\text { Reverse: TATGGTTGTTCACGACTCCTTCAC }\end{array}$ & $73 \mathrm{bp}$ \\
\hline hsa-miR-203a-3p & $\begin{array}{l}\text { Forward: CGATGCTGTGAAATGTTTAGGAC } \\
\text { Reverse: TATGGTTTTGACGACTGTGTGAT }\end{array}$ & $67 \mathrm{bp}$ \\
\hline hsa-miR-99a-5p & $\begin{array}{c}\text { Forward: CATTACTAAACCCGTAGATCCGAT } \\
\text { Reverse: TATGGTTTTGACGACTGTGTGAT }\end{array}$ & $67 \mathrm{bp}$ \\
\hline hsa-miR-106b-5p & $\begin{array}{l}\text { Forward: AAATGCTCATAAAGTGCTGACAGT } \\
\text { Reverse: TATGGTTTTGACGACTGTGTGAT }\end{array}$ & $66 \mathrm{bp}$ \\
\hline hsa-miR-2I-5p & $\begin{array}{l}\text { Forward: ACGTTGTGTAGCTTATCAGACTG } \\
\text { Reverse: AATGGTTGTTCTCCACACTCTC }\end{array}$ & $74 \mathrm{bp}$ \\
\hline hsa-miR-19b-3p & $\begin{array}{l}\text { Forward: TGTCATAATCACTGTGCAAATCC } \\
\text { Reverse: TATGGTTTTGACGACTGTGTGAT }\end{array}$ & $62 \mathrm{bp}$ \\
\hline hsa-miR-22-5p & $\begin{array}{l}\text { Forward: CAATCAACGAAGTTCTTCAGTGG } \\
\text { Reverse: TATGCTTGTTCTCGTCTCTGTGTC }\end{array}$ & $71 \mathrm{bp}$ \\
\hline U6 & $\begin{array}{c}\text { Forward: CAGCACATATACTAAAATTGGAACG } \\
\text { Reverse: ACGAATTTGCGTGTCATCC }\end{array}$ & $76 \mathrm{bp}$ \\
\hline
\end{tabular}

incubate the cell climbing slices overnight at $4^{\circ} \mathrm{C}$. The primary antibody was then removed and fresh PBS was added. After washing with PBS three times, climbing slices were incubated with the secondary antibody goat anti-rabbit IgG (1:500, Abbkine, Hubei, China) and protected from light for $1 \mathrm{~h}$. The nuclei were counterstained with 4',6-diamidino-2-phenylindole (DAPI) (1:500) for 5 min. After the final washing steps in PBS $(3 \times 10 \mathrm{~min})$, the images were captured under an upright fluorescence microscope (Leica, Berlin, Germany).

\section{Dual-Luciferase Reporter Assay}

The fragments of wild-type 3'-UTR and the miR-1246 "seed" mutant 3'-UTR of POSTN were synthesized and then inserted into pmirGLO luciferase reporter plasmids, which were named as POSTN-WT and POSTN-MUT. The 293 T cells were co-transfected with a recombinant luciferase reporter plasmid (WT or MUT plasmids) and miRNA (NC or
miR-1246 mimic) via Lipofectamine 2000. After $48 \mathrm{~h}$, the relative luciferase activity was assessed by the DualLuciferase Reporter Assay kit (Beyotime, Shanghai, China).

\section{Statistical Analysis}

A ROC curve based on the miRNA expression in the samples was plotted to analyze the sensitivity, specificity, and cutoff value of miRNAs, and the AUC was calculated in order to evaluate the diagnostic performance of the screened miRNAs as biomarkers. All results were expressed as mean \pm standard deviation; differences between two groups were analyzed using Student's $t$-test. A two-tailed $\mathrm{p}$ value $<0.05$ was considered statistically significant.

\section{Results}

\section{Identification of DE-miRNAs}

An $|\mathrm{FC}|>1.5$ and a $\mathrm{p}$ value $<0.05$ were considered as criteria for screening the DE-miRNAs. A remarkably 
different miRNA expression pattern was noted in the airway epithelium in normal subjects and patients with asthma (Figure 1A and B). Among these, 83 DEmiRNAs - including 37 down-regulated and 46 upregulated miRNAs - were found in the GSE25230 profile (Figure 1A), whereas 209 DE-miRNAs, including 118 upregulated and 91 down-regulated miRNAs, were found in the GSE142237 profile (Figure 1B).

The candidate DE-miRNAs were generated from the two intersected datasets through a Venn diagram (Figure 1C). Detailed information on intersecting DEmiRNAs is shown in Table 2, including four downregulated (miR-203a-3p, miR197-3p, miR-92b-3p, and miR-193a-5p) and two up-regulated miRNAs (miR-124$5 \mathrm{p}$ and miR-1246), which were speculated to participate in the onset and development of asthma (Figure 1D).

\section{Diagnostic Value of Selected miRNAs in Asthma}

To investigate the efficacy of DE-miRNAs as potential biomarkers in asthma, we performed a ROC curve analysis using $\mathrm{R}$ software (version 3.6.2). The area under the curve (AUC) represents the potential of the DE-miRNAs as diagnostic markers of these six miRNAs (Figure 1E-J) as follows: miR-92b-3p (AUC $=0.764)$, miR-203 (AUC $=0.727), \mathrm{miR}-124-5 \mathrm{p}(\mathrm{AUC}=0.661), \mathrm{miR}-193 \mathrm{a}-5 \mathrm{p}(\mathrm{AUC}$ $=0.764)$, miR-197-3p (AUC $=0.794)$ and miR-1246 $(\mathrm{AUC}=0.836)$. Collectively, the results revealed that these miRNAs, especially miR-1246, had excellent diagnostic efficiency for distinguishing patients with asthma and healthy subjects.

\section{miRNA-Target Gene Interactions}

Next, TargetScan, miRWalk, and TarBase were used to predict the target genes of screened miRNAs. As shown in Figure 2A, the network of miRNA-mRNA interactions was visualized in Cytoscape.

\section{GO Annotation and KEGG Pathway Analysis of Predicted Target Genes}

GO annotation and KEGG pathway enrichment analysis of all 1373 target genes were performed by the DAVID online tool. The results of the GO biological process (BP) analysis indicated that the target genes of DEmiRNAs were primarily enriched in transcription DNAtemplated, regulation of transcription DNA-templated, positive regulation of RNA polymerase II promoter, positive regulation of transcription, and DNA-templated and negative regulation of RNA polymerase II promoter. In addition, the nucleus, cytoplasm, and plasma membrane accounted for the majority of the cellular component (CC) category. In molecular function, the target genes were mainly enriched in protein binding, ATP binding, and DNA binding (Figure S1A).

KEGG pathway analysis showed that the target genes of the DE-miRNAs were significantly enriched in the cAMP signaling pathway, focal adhesion, and the cGMPPKG signaling pathway (Figure S1B).

\section{PPI Network}

The PPI network of the Top 100 linked target genes of DEmiRNAs was analyzed via the STRING database and visualized by Cytoscape software, with 100 nodes and 603 edges, as shown in Figure 2B. The top 10 genes were hub genes with a high-ranking degree and significantly closely associated with the miRNAsmarked orange and linked with much larger circles in Figure 2B.

\section{Identification of DE-mRNAs in Asthma}

DE-mRNAs were identified in the GSE43696 after standardizing the microarray data (using the preprocess Core package in $\mathrm{R}$ software), including 124 up-regulated genes and 143 down-regulated genes (Figure 2C). The DE-mRNAs were further visualized in a heatmap (Figure 2D). All samples used to detect differences in miRNA and mRNA expression were derived from airway biopsy tissue. The intersection of the target genes of DE-miRNAs and DEmRNAs are shown in a Venn diagram, which revealed that 31 genes were common in the predicted genes of DEmiRNAs and real DE-mRNAs (Figure 2E).

\section{Interactions Between DE-miRNAs and DE-mRNAs}

The relationship between DE-miRNAs and DE-mRNAs is shown in Figure 2F. These six DE-miRNAs are likely to affect the pathogenesis of asthma by altering the expressions of these 31 differentially expressed genes in the airways of patients with asthma.

\section{Characterizing the Functions of miRNAs in Airway Remodeling/EMT and Eosinophilic Inflammation Cellular Model} To further explore the functions of these screened miRNAs as well as several other miRNAs of interest 
A

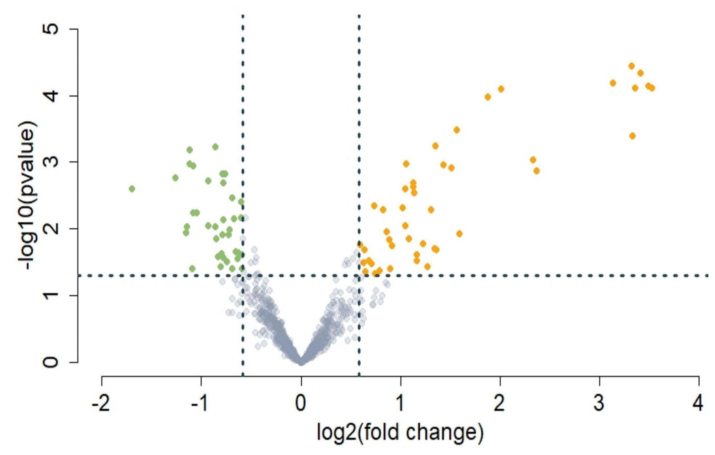

C

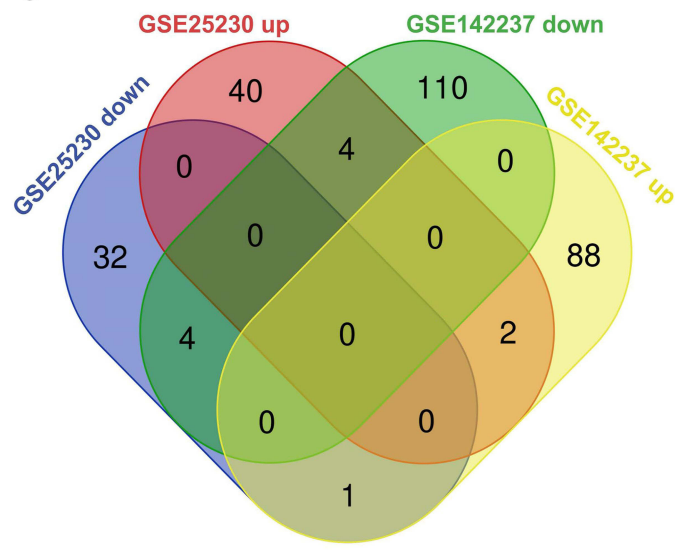

D

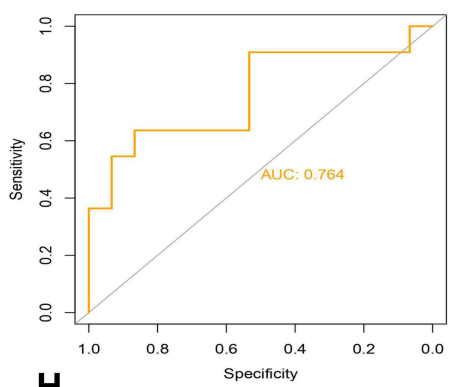

H

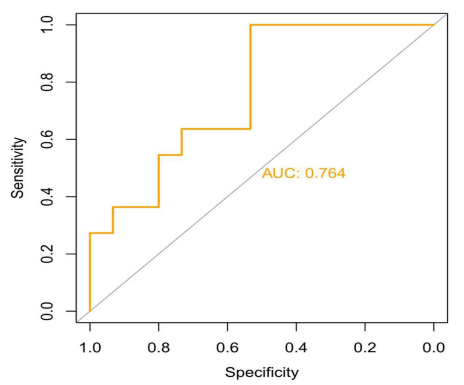

F
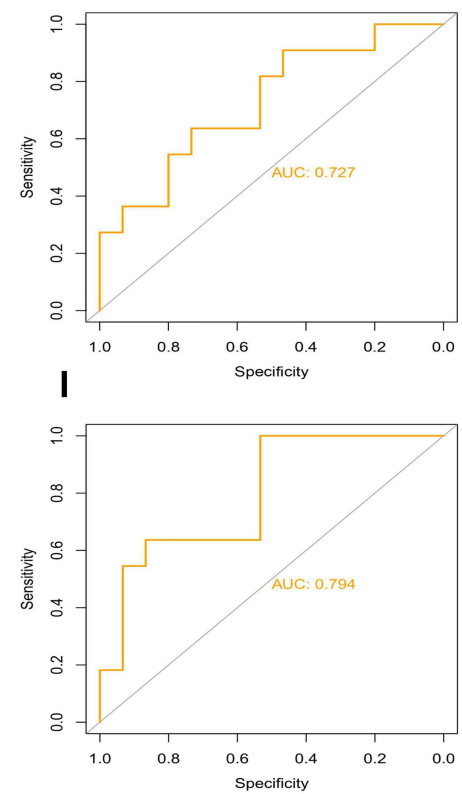

B

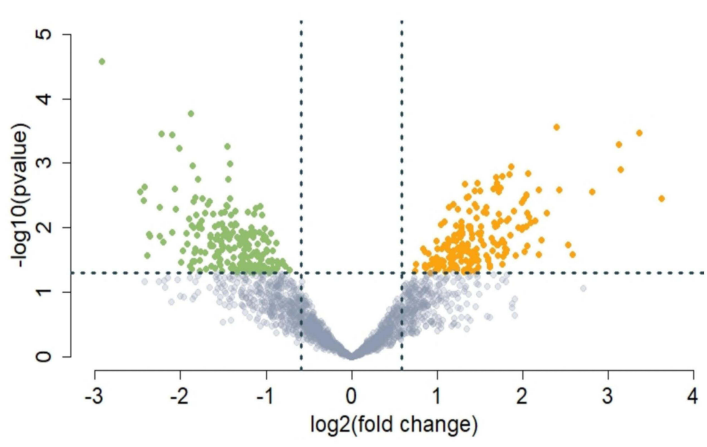

D

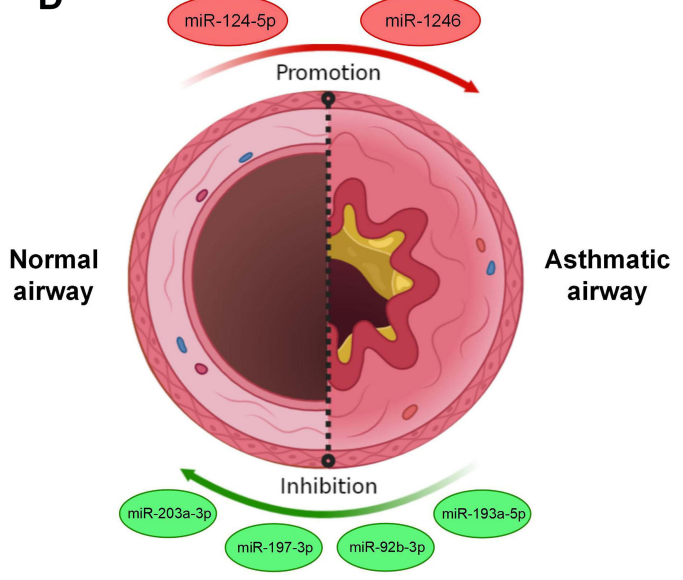

G
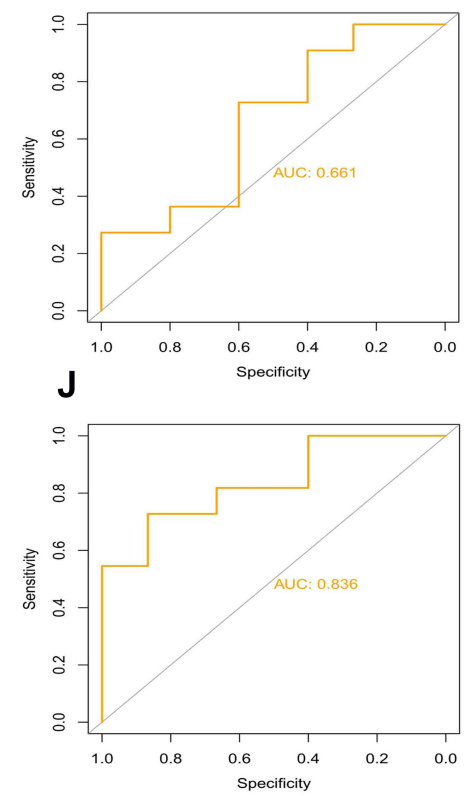

Figure I Identification of differentially expressed (DE)-miRNAs. (A and B) Volcano plot of differentially expressed miRNAs in two datasets. Many miRNAs are differentially expressed based on analysis of the GSE25230 and GSE.The red dots represent up-regulated miRNAs and the green dots represent down-regulated miRNAs. |FC| > I.5 and a $p$ value $<0.05$ are set as the screening criterion (C) Venn diagram of four series. The intersection represents the common DE-miRNAs, the results of which revealed that there were five intersecting miRNAs, including miR-92b-3p, miR-203, miR-124-5p, miR-193a-5p, miR-197-3p, and miR-I246 (D) presentation of identified miRNAs associated with asthma. The red circles represent up-regulated miRNAs and the green circles represent down-regulated miRNAs. (E-J) ROC curve analysis was performed on screened miRNAs using the package pROC. ROC curve of miR-92b-3p (AUC = 0.764, accuracy $=0.692)$, miR-203 (AUC =0.727, accuracy =0.73I), miR-124-5p (AUC = $0.66 \mathrm{I}$, accuracy $=0.654)$, miR-193a-5p $(A \cup C=0.764$, accuracy $=0.731)$, miR-197-3p $(A \cup C=0.794$, accuracy $=0.731)$, and miR-1246 $(A \cup C=0.836$, accuracy $=0.808)$. Abbreviations: AUC, area under the receiver operating characteristic curve; $\mathrm{Cl}$, confidence interval. 
Table 2 miRNA Identified from Two miRNA Datasets

\begin{tabular}{|l|c|c|c|}
\hline Symbol & P value (GSE25230/GSE 1 42237) & logFC (GSE25230/GSE I42237) & Trend \\
\hline hsa-miR-197-3p & $0.0103427 / 0.0131059$ & $-0.7203174 /-1.025878$ & down \\
hsa-miR-124-5p & $0.0170273 / 0.0098896$ & $0.5885927 / 1.945912$ & up \\
hsa-miR-1246 & $0.0298949 / 0.0016051$ & $1.1596158 / 1.756827$ & up \\
hsa-miR-92b-3p & $0.0305739 / 0.0495062$ & $-0.7424665 /-0.963976$ & down \\
hsa-miR-193a-5p & $0.0369103 / 0.0047935$ & $-0.8075554 /-2.247316$ & down \\
hsa-miR-203a-3p & $0.0393509 / 0.0477603$ & $-1.0938027 /-1.338249$ & down \\
\hline
\end{tabular}

(miR-22-5p, miR-19b-3p, miR-24-5p, miR-99a-5p, and miR-106b-5p), we constructed two commonly employed cell models of asthma (airway remodeling/ EMT cellular model induced by TGF- $\beta 1$ and eosinophilic inflammation cellular model induced by IL-13) and examined the expression level of these screened miRNAs. We also validated the expression of the internal reference gene U6 which was not altered upon TGF$\beta 1 /$ IL-13 treatment at experimental concentration and confirmed it as a stable reference gene (Figure S2). The results from the eosinophilic inflammation cellular model revealed that the miRNA levels of miR-92b-3p, miR-1246, miR-197-3p, and miR-124-5p were significantly highly expressed in the IL-13 stimulated group while the expression of other miRNAs were constant (Figure 3A). These findings suggest that these four miRNAs may play a role in eosinophilic inflammation that is an indication of asthma.

Subsequently, to evaluate the roles of these miRNAs in airway remodeling, another key feature of asthma, we constructed an airway remodeling/EMT cellular model using human recombinant protein TGF- $\beta 1$. After stimulation with TGF- $\beta 1$, we observed that the morphology of BEAS-2B cells changed gradually from normal to long, spindle, fibroblast-like cells with increasing stimulation time (Figure 3B). The expression of remodelingassociated genes (MMP-9, collagen, $\alpha$-SMA, and fibronectin) and EMT-associated genes (E-cadherin, N-cadherin, and vimentin) was significantly altered as shown by RTPCR and immunofluorescence (Figure 3C and D). Taken together, this shows that the models we constructed were successful. Concurrently, we found significantly altered expression of miR-197-3p, miR-193a-5p, miR-1246, miR-21-5p, and miR-92b-3p in this process (Figure 3E). Similar findings occurred after stimulating BEAS-2B cells with a high concentration TGF- $\beta 1$ (20 ng/mL), which demonstrates the high repeatability of the results (Figure 3E).

Given that IL-13 and TGF- $\beta$ are two key regulators in initiating asthma, these results suggest that these differentially expressed miRNAs play a key role in regulating asthma development. In order to further obtain valuable miRNAs from these we screened, we also collected blood samples collected from 10 patients with asthma and 10 healthy subjects (Table $\mathrm{S} 1$ and $\underline{\mathrm{S} 2}$ ). The miRNAs assay workflow of included settings and processes were shown in Figure 4A. By detecting the level of selected miRNAs in blood, we found a significant difference in miR-92b-3p, miR-1246, miR203a-3p, miR-21-5p, and miR-19b-3p levels among the groups, further supporting the role of these miRNAs in asthma development and progression (Figure 4).

\section{POSTN is a Target of miR- 1246}

Interestingly, after integrated analysis of all the above results, we noticed that the expression of miRNA-1246 is prominent in the blood sample of patients with asthma, which was consistent with the results of bioinformatics analysis of data from GSE25230 and GSE142237 datasets. These results aroused our interest in exploring the role of miR-1246 during asthma pathogenesis. Initially, miRNA-FAM (stable negative control conjugated with FAM), a miR-1246 mimic, and inhibitor transfection efficiency were assayed via qRT-PCR and immunofluorescence. As shown in Figure 5A-C, BEAS2B cells grew well after transfection, with high transfection efficiency.

Using bioinformatics analysis, we predicted that miR1246 could bind to POSTN, THSD7A, SIAH3, DNAJC12, and $C A D M 2$, five miR-1246 targeted genes with relatively high potential derived from the screening study described above (Figure 5). However, in our subsequent experiments, the levels of THSD7A, SIAH3, CADM2, and DNAJC12 in BEAS-2B cells transfected with miR-1246 mimic or 

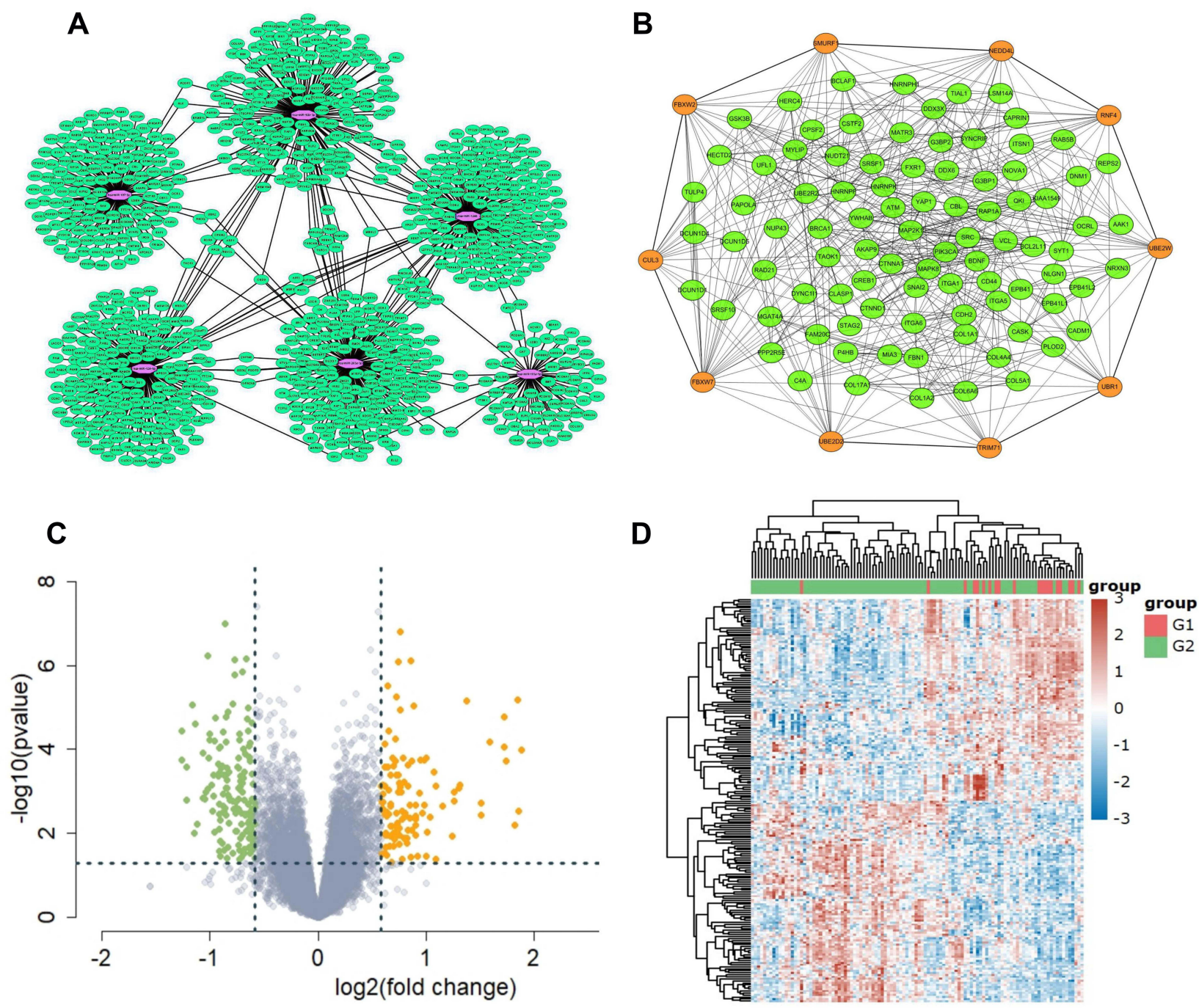

$\mathbf{E}$

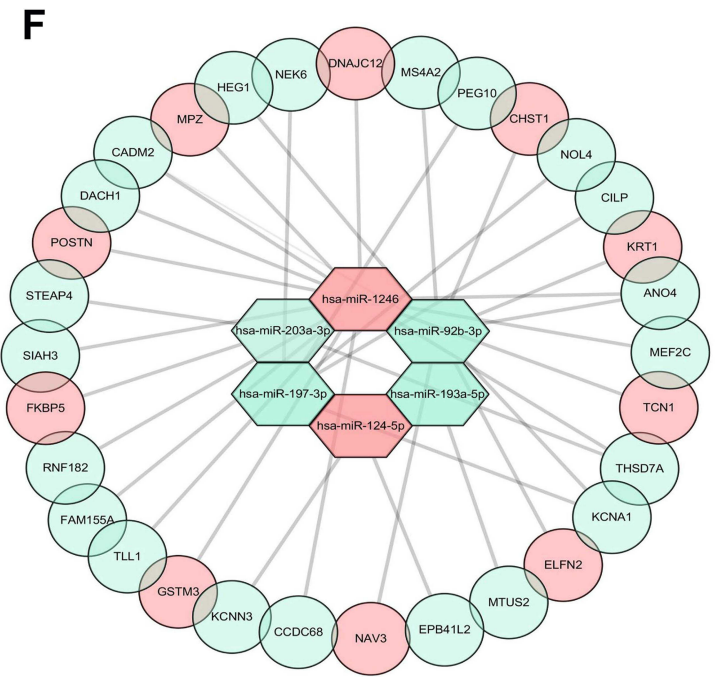

Figure 2 (A) Construction of epithelial miRNA-mRNA regulatory network in asthma. Interaction networks of miRNAs and their predicted target genes. The purple dots represent miRNAs and the green dots represent target mRNAs. (B) PPI network of the target genes constructed with Cytoscape software. Orange dots represent the key Hub genes of Top 10. (C and D) The DE-mRNAs in airway epithelium in normal controls and patients with asthma are presented with a volcano plot, and a heat map was created to show the hierarchical clustering analysis of different expression patterns. The red dots represent up-regulated mRNAs and the green dots represent down-regulated mRNAs. $|\mathrm{FC}|>1.5$ and a p value $<0.05$ are set as the screening criterion (E) Venn diagram showing intersection of DE-mRNAs and target genes of identified miRNAs. The intersected region represents the intersection results and 31 genes were intersected in the two groups including 12 genes up-regulated (red) and 19 genes down-regulated (light green) in asthmatics airway epithelium (F) the MiRNA-mRNA regulatory network during asthma progression based on six screened miRNAs and 31 mRNAs. Up-regulated genes are marked in red; down-regulated genes are marked in light green. 

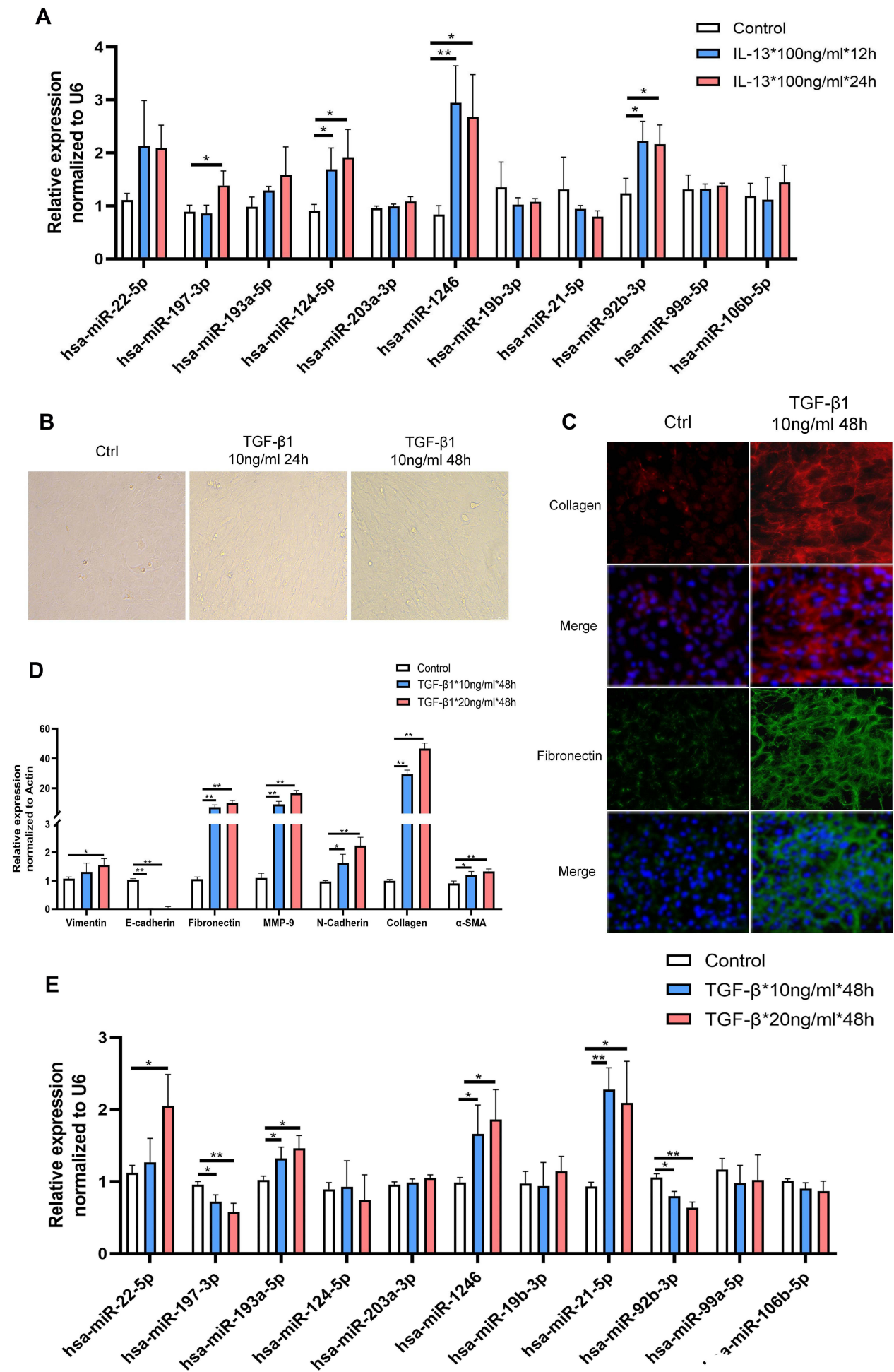

Figure 3 The level of identified miRNAs in asthma-related cellular models. (A) The level of identified miRNAs in IL-I3-induced BEAS-2B cells at various concentrations. (BD) Quantitative reverse transcription-PCR ( $q$ RT-PCR) and morphological and immunofluorescence analyses were commonly used to confirm that an airway remodeling/ epithelial mesenchymal transition (EMT) cellular model induced by TGF- $\beta$ I was successfully constructed. (E) The level of identified miRNAs in TGF- $\beta$ I-induced BEAS-2B cells at various concentrations. Data are represented as means \pm SEMs from 3 independent experiments. $* \mathrm{P}<0.05 ; * * \mathrm{P}<0.01$.

inhibitor were confounding, which indicated that the interactions between miR-1246 and these four genes might be complicated and involve a variety of mechanisms.
We also found that the expression of POSTN, a key immunological biomarker of asthma, was decreased when the miR-1246 mimic was transfected and began 


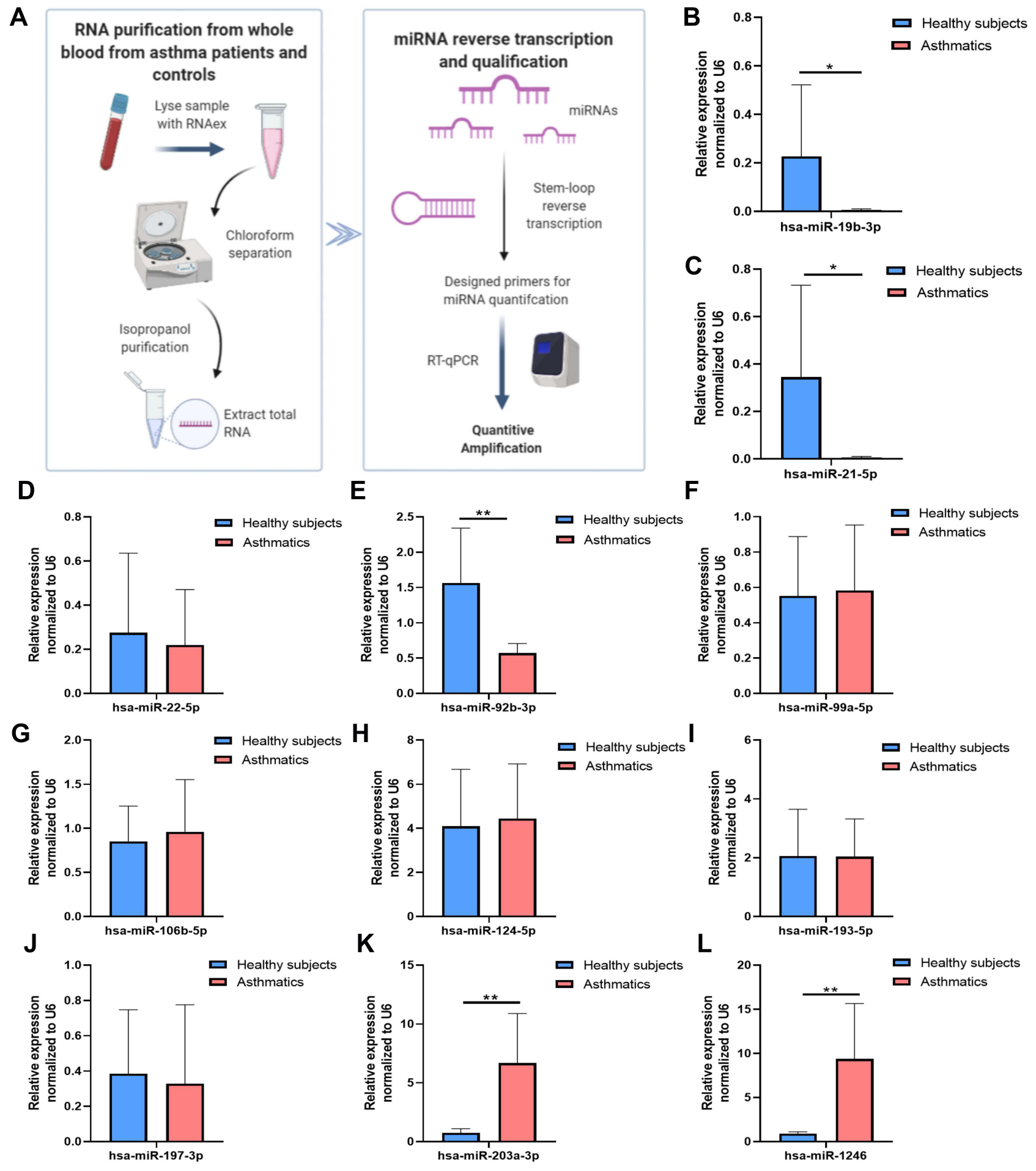

Figure 4 DE-miRNAs in blood samples in patients with asthma and healthy controls. (A) A flow chart for quantification of the selected miRNAs in blood (B) hsa-miR-19b3p (C) hsa-miR-2I-5p (D) hsa-miR-22-5p (E) hsa-miR-92b-3p (F) hsa-miR-99a-5p (G) hsa-miR-I06b-5p (H) hsa-miR-I24-5p (I) hsa-miR-I93a-5p. (J) hsa-miR-I97-3p (K) hsa-miR-203a-3p (L) hsa-miR-1246. Data are represented as means \pm SEMs from 3 independent experiments. $* P<0.05$; $* * P<0.0$ I.

trending upward when the miR-1246 inhibitor was transfected (Figure 5H). Furthermore, the dual-luciferase reporter gene assay found that after co-transfection with miR-1246 mimics, the luciferase activity of the WT 3 '-
UTR reporter gene significantly decreased, whereas the luciferase activity of the mutant reporter gene was not affected $(P<0.01)$. Thus, we suggest that POSTN is one of the genes targeted by miR-1246. 
A

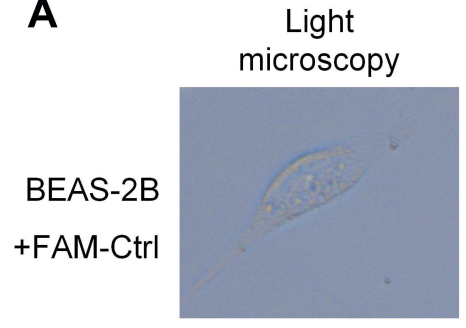

D

SIAH3 3' UTR 5' ..CAGUGACGGAAAUCCAUA.. hsa-miR-1246 3' ACGAGGUUUUUAGGUAA
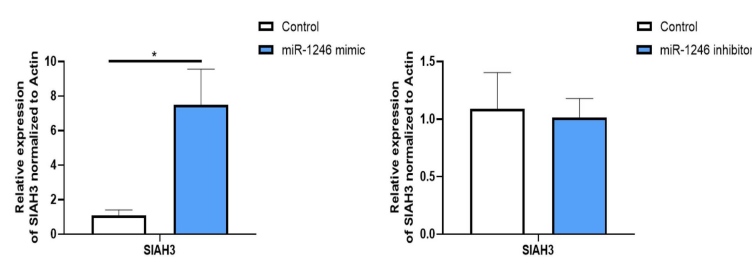

$\mathbf{F}$

THSD7A 3' UTR 5' ...GCAGUGUUAAUCCAU. | | | | | | hsa-miR-1246 3' ACGAGGUUUUUAGGUAA
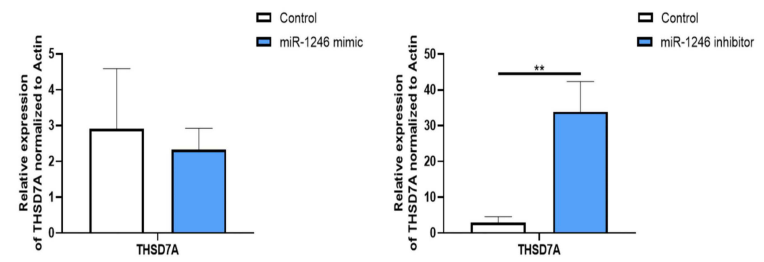

H
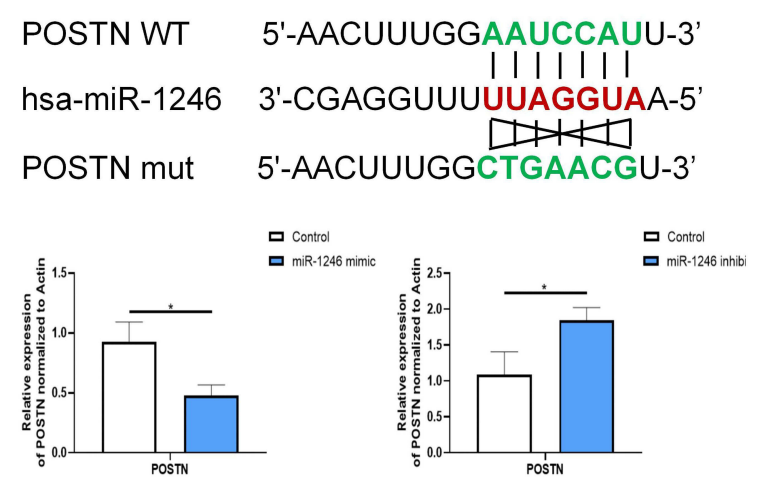

B

C
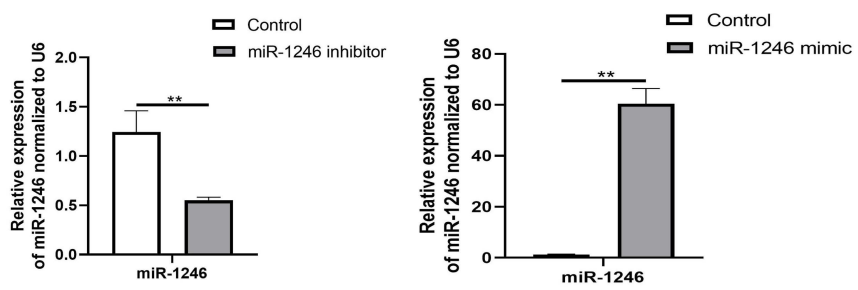

E

CADM2 3' UTR 5' ...UUACUGUACCAUCCAUA..

| | | | | |

hsa-miR-1246 3' ACGAGGUUUUUAGGUAA
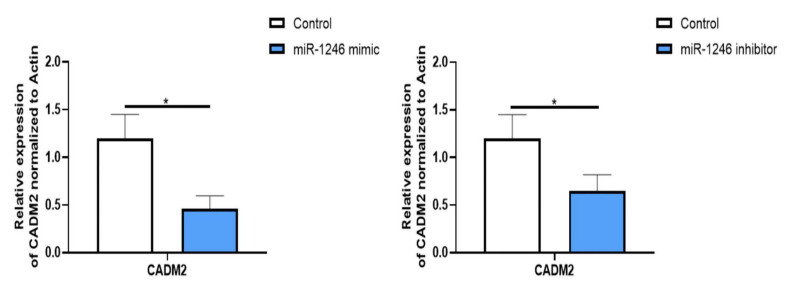

G

DNAJC12 3' UTR 5' ...CUUAUAGAAUCCAU.

| | | | | | |

hsa-miR-1246 3' ACGAGGUUUUUAGGUAA
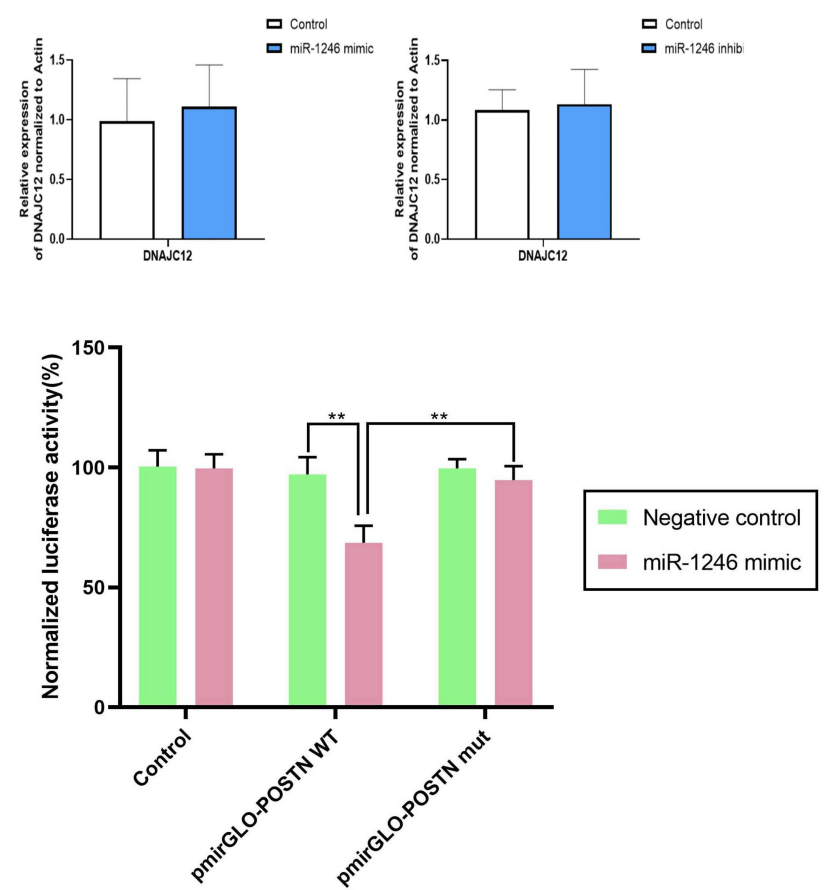

Figure 5 POSTN was a direct target of miR-1246. (A-C) The transfection efficiency was assessed using qRT-PCR and immunofluorescence analysis. (D-H) Five DE-genes (POSTN, THSD7A, SIAH3, DNAJCI 2, and CADM2) that have relatively high prediction-targeted scores were predicted to interact with miR-I246 using TargetScan and validated by qRT-PCR and the dual-luciferase reporter assay using miR-1246 mimics/inhibitors. Data are represented as means \pm SEMs from 3 independent experiments. $* P<0.05$; $* * P<0.01$.

\section{MiR-I246 Down-Regulated POSTN Expression and Affected Airway Remodeling in in vitro Models}

According to previous studies, IL-13 is considered to be an essential factor in the induction of periostin. Thus, to further substantiate our findings, we determined the optimal IL-13 stimulation duration to induce the production of periostin (Figure 6A and B). Strikingly, following IL-13 stimulation for an appropriate time (approximately $12 \mathrm{~h}$ ), the effect of miR-1246 on POSTN became more 


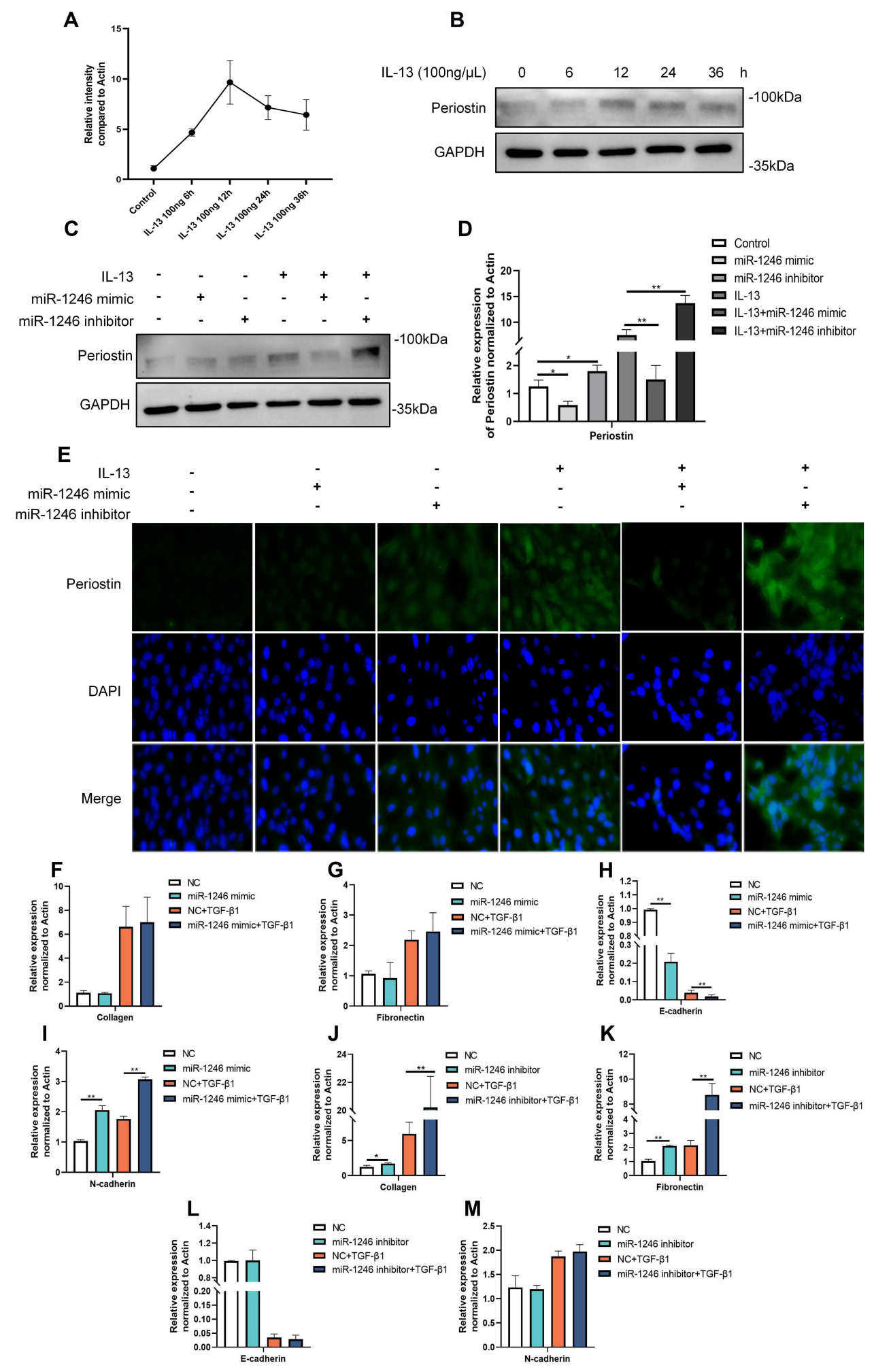

Figure 6 Effects of miR-1246 on POSTN and remodeling. (A) Western blot and qRT-PCR assays were performed to determine the full time-response curve of periostin. The maximum response was observed with $100 \mathrm{ng} / \mathrm{mL} I L-13$ at $12 \mathrm{~h}$. (C-E) The expression of POSTN in BEAS-2B cells transfected with the miR-1246 mimic, miR-I246 inhibitors, or miR-control was measured by Western blot, RT-PCR and immunofluorescence analysis. (F-M) RT-PCR was used to examine the effects of miR-1246 on airway remodeling and EMT using miR-1246 mimics (F-I) and inhibitors (J-M). Similar results were obtained in three independent experiments. *P < $0.05 ; * * \mathrm{P}<0.01$. 
pronounced (Figure 6C-E). The Western blot and RTqPCR data were also confirmed by immunofluorescence staining, which all suggested that POSTN was the target of miR-1246 (Figure 6C-E). Because of undesired effects after anti-IL-13 treatment (lebrikizumab), clinical applications of periostin as a proposed systemic biomarker of airway eosinophilia in asthma have been greatly limited. In this study, our data may compensate for this deficiency.

We also analyzed the effects on miR-1246-regulated gene expression in remodeling and EMT using quantitative PCR, and significant effects were also found (Figure 6FM). Collectively, all the results suggest that miR-1246 may play an intricate role in asthma progression and be a potential target in the treatment and diagnosis of asthma.

\section{Discussion}

According to the updated GINA, asthma, which exhibits varying symptoms with variable expiratory flow limitations, is defined as a heterogeneous clinical syndrome rather than a single disease entity. ${ }^{12}$ As stated above, efficient methods for asthma identification and classification are lacking, severely restricting the implementation of individualized precision therapy. To date, there has been no single diagnostic biomarker for asthma. The airway epithelium provides first-line host defenses against invading pathogens and can be directly obtained from bronchoscopies and cultures ex vivo. ${ }^{13}$ There has been ample evidence in the literature that the airway epithelium is an essential controller of bronchial inflammation, remodeling, and hyperreactivity clinically relevant to asthma. ${ }^{14}$ Thus, strategies targeting the airway epithelial cells may arrest the pathologic progression of asthma and decrease its burden on patients. ${ }^{14}$ However, the mechanisms underlying the airway epithelial cell dysfunction in asthma remain largely unknown. ${ }^{15}$

The discovery of miRNA in $1993^{16}$ made it possible to obtain a more comprehensive understanding of the asthma pathophysiology. Concurrently, it also provides a fundamental basis for the selection of strategies for precise diagnosis and molecular-based surrogate classification for asthma. ${ }^{17}$ Increasing evidence has shown that varying numbers of miRNAs (including miR-19, miR-21, miR 22, miR141, miR221, and miR-455) can affect multiple aspects of the pathogenesis of asthma, such as aggravating airway remodeling, regulating mucus production, and affecting $\mathrm{T}$ helper cell/cytokine imbalance. ${ }^{18,19}$ In recent studies, levels of miRNAs (miR-92b, miR-210, and miR-34a) in extracellular vesicles secreted from airway epithelial cells were altered and associated with asthma development. ${ }^{20}$
However, as described above, even with an increasing number of identified miRNAs that have been found to function in maintaining airway homeostasis, the miRNA expression signatures in airway epithelium during asthma progression, are still undetermined.

In this study, we constructed a miRNA-dominated regulatory network in the airway epithelium samples of patients with asthma. DE-miRNAs in the GSE25230 and GSE142237 datasets were screened in samples from patients with asthma and in normal biopsy samples, and we finally selected six collective DE-miRNAs as "plausible" DEmiRNAs. Based upon ROC curve analysis, we further calculated the AUC and quantified the diagnostic availability of these six miRNAs. Through three online prediction tools, TargetScan (version 7.2), miRWalk (version 2.0), and TarBase (version 7.0), an miRNAs-mRNAs interaction network of these miRNAs was constructed. In addition, GO and KEGG enrichment analyses of the mRNAs in the miRNA network were performed. To further identify the functional target genes during asthma progression, another assay dataset, GSE43696, was used to analyze the difference of gene expression profiles between the airway epithelium in patients with asthma and healthy controls. Through combined analysis, a more value-based miRNA-mRNA network was constructed, including six screened miRNAs and 31 targeted genes. This integrative mRNA-miRNA network and the subsequent functional validation studies in vitro provide some evidence for a causal relationship between regulatory miRNAs and asthma-associated gene expression.

In recent years, periostin has emerged as a promising and viable biomarker of asthma, especially in eosinophilic asthma. $^{21}$ It is also widely acknowledged to participate in abnormal airway epithelial function in asthma, including promoting mucin and remodeling-related genes expression. $^{22,23}$ Some studies, although based on small sample size, have suggested a correlation between the serum periostin and reduced lung function (airway remodeling) and airway hyper-responsiveness (AHR). ${ }^{24-26}$ Despite these strengths, several key shortcomings limit the prognostic usefulness of periostin. Currently, in some certain conditions (eg, after IL-13 targeted therapy), the association between periostin levels and eosinophil numbers became nonsignificant. ${ }^{27}$ Furthermore, in a prospective, real-world study, researchers enrolled 465 eligible patients with severe asthma and examined the relationship between asthma exacerbation risks and the serum periostin level. Regrettably, in this year-long longitudinal study, researchers did not find any prognostic value of serum periostin in detecting the rate of asthma 
exacerbations. ${ }^{28}$ These findings, coupled with some other data, have greatly hindered the application of periostin in the clinic. ${ }^{22,29-31}$ Our research found that during the asthma progression, miR-1246 not only changes dynamically but also controls the expression of periostin both at the transcriptional and protein levels. These effects become more pronounced as IL-13 stimulates periostin expression under such appropriate conditions as time and concentration. Given the interaction between miR-1246 and periostin in patients with asthma, measurement combined with these two factors may, to some extent, reduce underlying influences from confounding factors and serve as a potential strategy for evaluating patients. We are currently planning studies to explore this scenario and further validate the hypothesis in subsequent experiments.

We have to admit that there are several limitations of this study. Although we minimized $\backslash$ the likelihood of potential sources of bias as much as possible, we did not analyze the data from the arrays originally. Therefore, the presence of some confounding factors like batch effects or biological differences are unavoidable, and the results for this study should be interpreted cautiously. In addition, miR-1246 is absent in mouse which limits research on its roles in in vivo models, and further work would be required to dissect the function of the miR-1246 in asthma.

\section{Conclusion}

We constructed a preliminary integral miRNA-mRNA regulatory map in asthmatic airway epithelium based on six screened DE-miRNAs and 31 targeted genes. We further explored their functions in two cellular models and analyzed the diagnostic potential of these screened miRNAs in blood samples. Our experiments provide further support for crafting the architecture for the asthmatic airway epithelium microenvironment to seek precise therapeutic targets. In addition, miRNA-1246, confirmed to be up-regulated by stimulation of two causative factors in asthma, could also regulate the expression of POSTN. In light of the essential regulatory role of periostin in asthma progression, targeting miR1246 may be a promising therapeutic target for asthma. Furthermore, detection of periostin and miR-1246 in combination could be a valuable prognostic and diagnostic marker as compared with a single marker. However, the miR-1246 was not present in mice, limiting in-depth research on its role in asthma.

\section{Abbreviations}

AUC, area under the curve; BPs, biological processes; CCs, cellular components; DAVIDs, Database for Annotation, Visualization and Integrated Discovery; DE, differentially expressed; EMT, epithelial mesenchymal transition; FDR, false discovery rate; GEO, Gene Expression Omnibus; GO, Gene Ontology; IL, interleukin; KEGG, Kyoto Encyclopedia of Genes and Genomes; MFs, molecular functions; miRNA, microRNA; PPI, proteinprotein interaction; ROC, Receiver operating characteristics; RT-qPCR, Reverse transcription quantitative PCR; TGF, transforming growth factor.

\section{Data Sharing Statement}

The data used and analyzed during this research are available from the corresponding author upon reasonable request.

\section{Ethics Approval and Consent}

The study was approved by the ethical committee of Qianfoshan Hospital Affiliated to Shandong University (Ethics review number: 2021-S923). All patients provided informed consent to participate and for the data to be published.

\section{Consent for Publication}

Written informed consent for publication was obtained from all participants.

\section{Author Contributions}

All authors made a significant contribution to the work reported, whether that is in the conception, study design, execution, acquisition of data, analysis and interpretation, or in all these areas; took part in drafting, revising or critically reviewing the article; gave final approval of the version to be published; have agreed on the journal to which the article has been submitted; and agree to be accountable for all aspects of the work.

\section{Funding}

This work was supported by grants from the National Natural Science Foundation of China (81770029). The funders had no role in the study design, data collection, data analysis, or preparation of the manuscript.

\section{Disclosure}

The authors declare that they have no conflicts of interest for this work. 


\section{References}

1. Zhang J, Dong L. Status and prospects: personalized treatment and biomarker for airway remodeling in asthma. J Thorac Dis. 2020;12 (10):6090-6101. doi:10.21037/jtd-20-1024

2. Bonato M, Gallo E, Bazzan E, et al. Air pollution relates to airway pathology in wheezing children. Ann Am Thorac Soc. 2021. doi:10.1513/AnnalsATS.202010-1321OC

3. Abu-Kishk I, Polakow-Farkash S, Elizur A. Long-term outcome after pediatric intensive care unit asthma admissions. Allergy Asthma Proc. 2016;37(6):169-175. doi:10.2500/aap.2016.37.4008

4. Arshad S, Hodgekiss C, Holloway J, et al. Association of asthma and smoking with lung function impairment in adolescence and early adulthood: the Isle of Wight Birth Cohort Study. Eur Respir J. 2020;55(3):1900477. doi:10.1183/13993003.00477-2019

5. Chiu C, Huang M. Asthma in the precision medicine era: biologics and probiotics. Int J Mol Sci. 2021;22(9):4528. doi:10.3390/ijms22094528

6. Hewitt R, Lloyd C. Regulation of immune responses by the airway epithelial cell landscape. Nat Rev Immunol. 2021;21:347-362. doi:10.1038/s41577-020-00477-9

7. Stefano L, Rössler OG, Griesemer D, Hoth M, Thiel G. P2X(7) receptor stimulation upregulates Egr-1 biosynthesis involving a cytosolic $\mathrm{Ca}(2+)$ rise, transactivation of the EGF receptor and phosphorylation of ERK and Elk-1. J Cell Physiol. 2007;213 (1):36-44. doi:10.1002/jcp. 21085

8. Akdis C. Does the epithelial barrier hypothesis explain the increase in allergy, autoimmunity and other chronic conditions? Nat Rev Immunol. 2021. doi:10.1038/s41577-021-00538-7

9. Incorvaia C, Al-Ahmad M, Ansotegui I, et al. Personalized medicine for allergy treatment: allergen immunotherapy still a unique and unmatched model. Allergy. 2021;76(4):1041-1052. doi:10.1111/all.14575

10. Cushing L, Jiang Z, Kuang P, Lü J. The roles of microRNAs and protein components of the microRNA pathway in lung development and diseases. Am J Respir Cell Mol Biol. 2015;52(4):397-408. doi:10.1165/rcmb.2014-0232RT

11. Szymczak I, Wieczfinska J, Pawliczak R. Molecular background of miRNA role in asthma and COPD: an updated insight. Biomed Res Int. 2016;2016:7802521. doi:10.1155/2016/7802521

12. Boulet L, Reddel H, Bateman E, Pedersen S, FitzGerald J, O'Byrne P. The Global Initiative for Asthma (GINA): 25 years later. Eur Respir J. 2019;54(2):1900598. doi:10.1183/13993003.00598-2019

13. Holgate ST, Davies DE, Lackie PM, Wilson SJ, Puddicombe SM, Lordan JL. Epithelial-mesenchymal interactions in the pathogenesis of asthma. J Allergy Clin Immunol. 2000;105(2 Pt 1):193-204. doi:10.1016/S0091-6749(00)90066-6

14. Lambrecht BN, Hammad H. The airway epithelium in asthma. Nat Med. 2012;18(5):684-692. doi:10.1038/nm.2737

15. Yang Y, Man J, Yingwei O, Adcock IM, Xin Y. Mechanisms and biomarkers of airway epithelial cell damage in asthma: a review. Clin Respir J. 2021;15:1027-1045. doi:10.1111/crj.13407

16. Lee RC, Feinbaum RL, Ambros V. The C. elegans heterochronic gene lin-4 encodes small RNAs with antisense complementarity to lin-14 Cell. 1993;75(5):843-854. doi:10.1016/0092-8674(93)90529-Y
17. Weidner J, Bartel S, Kılıç A, et al. Spotlight on microRNAs in allergy and asthma. Allergy. 2020;76(6):1661-1678.

18. Siddiqui S, Johansson K, Joo A, et al. Epithelial miR-141 regulates IL-13-induced airway mucus production. JCI Insight. 2021;6(5). doi:10.1172/jci.insight.139019.

19. Moheimani F, Koops J, Williams T, et al. Influenza A virus infection dysregulates the expression of microRNA-22 and its targets; CD147 and HDAC4, in epithelium of asthmatics. Respir Res. 2018;19 (1):145. doi:10.1186/s12931-018-0851-7

20. Bartel S, La Grutta S, Cilluffo G, et al. Human airway epithelial extracellular vesicle miRNA signature is altered upon asthma development. Allergy. 2020;75(2):346-356. doi:10.1111/all.14008

21. Jia G, Erickson R, Choy D, et al. Periostin is a systemic biomarker of eosinophilic airway inflammation in asthmatic patients. $J$ Allergy Clin Immunol. 2012;130(3):647-654.e610. doi:10.1016/j.jaci.2012.06.025

22. Burgess J, Jonker M, Berg M, et al. Periostin: contributor to abnormal airway epithelial function in asthma? Eur Respir J. 2021;57 (2):2001286. doi:10.1183/13993003.01286-2020

23. Izuhara K, Conway S, Moore B, et al. Roles of periostin in respiratory disorders. Am J Respir Crit Care Med. 2016;193(9):949-956. doi:10.1164/rccm.201510-2032PP

24. Inoue $\mathrm{T}$, Akashi $\mathrm{K}$, Watanabe $\mathrm{M}$, et al. Periostin as a biomarker for the diagnosis of pediatric asthma. Pediatr Allerg Immunol. 2016;27 (5):521-526. doi:10.1111/pai.12575

25. Mansur A, Srivastava S, Sahal A. Disconnect of type 2 biomarkers in severe asthma; dominated by $\mathrm{FeNO}$ as a predictor of exacerbations and periostin as predictor of reduced lung function. Respir Med. 2018;143:31-38. doi:10.1016/j.rmed.2018.08.005

26. Song J, You J, Jeong S, et al. Serum periostin levels correlate with airway hyper-responsiveness to methacholine and mannitol in children with asthma. Allergy. 2015;70(6):674-681. doi:10.1111/all. 12599

27. Hanania N, Korenblat P, Chapman K, et al. Efficacy and safety of lebrikizumab in patients with uncontrolled asthma (LAVOLTA I and LAVOLTA II): replicate, phase 3, randomised, double-blind, placebo-controlled trials. Lancet Respir Med. 2016;4(10):781-796. doi:10.1016/S2213-2600(16)30265-X

28. Buhl R, Korn S, Menzies-Gow A, et al. Prospective, single-arm, longitudinal study of biomarkers in real-world patients with severe asthma. J Allergy Clin Immunol Pract. 2020;8(8):2630-2639.e2636. doi:10.1016/j.jaip.2020.03.038

29. Licari A, Brambilla I, Sacchi L, Marseglia G, Ciprandi G. Periostin, type 2 biomarker, is not associated with asthma control grade in asthmatic allergic children. Respir Med. 2019;151:118-120. doi:10. 1016/j.rmed.2019.04.010

30. Castro-Rodriguez J, Atton I, Villarroel G, Serrano C. Serum periostin is not related to asthma predictive index. Allergologia et Immunopathol. 2018;46(3):235-240. doi:10.1016/j.aller.2017.05.012

31. Anderson H, Lemanske R, Arron J, et al. Relationships among aeroallergen sensitization, peripheral blood eosinophils, and periostin in pediatric asthma development. J Allergy Clin Immunol. 2017;139 (3):790-796. doi:10.1016/j.jaci.2016.05.033

Journal of Asthma and Allergy

Dovepress

\section{Publish your work in this journal}

The Journal of Asthma and Allergy is an international, peer-reviewed open-access journal publishing original research, reports, editorials and commentaries on the following topics: Asthma; Pulmonary physiology; Asthma related clinical health; Clinical immunology and the immunological basis of disease; Pharmacological interventions and

new therapies. The manuscript management system is completely online and includes a very quick and fair peer-review system, which is all easy to use. Visit http://www.dovepress.com/testimonials.php to read real quotes from published authors. 